\title{
Understanding Blockchain and Tokenomics
}

\author{
Lin William Cong* \\ Department of Finance, University of Chicago, USA \\ *Corresponding author: Lin William Cong, Department of Finance, University of Chicago, USA \\ Submission: 漈 May 24, 2018; Published: 些 June 07, 2018
}

\begin{abstract}
In this article, I review recent studies on blockchains, crypto currencies, and initial coin offerings. I organize the research into two broad categories on the economics of decentralized ledger technologies and their impacts on the real economy. I also outline promising directions for future research in this area.
\end{abstract}

Keywords: Blockchains; Tokenomics; Cryptography; Financial Cryptography.

\section{Introduction}

The advances in Fin Tech and sharing economy is largely driven by the increasing preference for forming peer-to-peer connections that are instantaneous and open, which is transforming how people interact, work, and consume. Yet financial and economic systems are often arranged around a series of centralized parties like banks, clearing house, and business behemoth. Blockchain-based crypto-applications are part of an attempt to resolve this issue by creating the financial architecture for peer-to-peer transactions and reorganizing society into a series of decentralized networks.

The blockchain technology is thus believed to have the potential to disrupt business and financial industry in a way similar to how the Internet revolutionized commerce and our daily lives. Yet the plethora of media discussions and industry propaganda are confusing and conflicting, to say the least.

To clarify, most societies and economies rely on consensus to function properly - protocols of behavior and state of the world that agents with divergent preferences and characteristics act upon as if they were the truth. Instead of having governments, courts, or third-party business arbitra- tors provide, oversee, and enforce the consensus, blockchains use a more decentralized manner of generating, storing, and distributing the record of rules and interactions. We have to recognize that even though the technology is new, the purpose is not alien, and the way the technology is used may not be as extreme as advocated in the media and in the industry. In particular, central bank blockchains and permissioned blockchains are examples of blockchain applications that are not fully decentralized, but many of the tradeoffs still apply.

As economists, our primary goal is not to advocate for the technology or engineer blockchain- based products for commercialization. In fact, the current generation of blockchain applications often fall short of its potential and ideal. Instead, we should aim to understand the economic tradeoffs and designs in realizing the technology's potential and functionalities, and how they impact the traditional markets and the real economy.

Given the key universal functionality of blockchains providing decentralized consensus, we follow Cong et al. [1] to argue that there are two economically relevant areas of research on blockchain:

(1) Blockchain mechanisms for generating and maintaining decentralized consensus, and

(2) Real-world implications given the functionality blockchain provides.

The first category can be further divided into the studies analyzing the general process of consensus generation for most blockchains, emphasizing the tradeoffs in decentralization, and the studies exploring the game theoretical topics including incentive provisions and market microstructure, taking as given a particular blockchain protocol such as the proof-of-work protocol in Bitcoin.

Computer scientists are among the earliest to study the underlying mechanism for generating decentralized consensus. Consensus algorithms and digital cash have preceded Bitcoin by many years, and Nakamoto's true innovation is to combine money with decentralized consensus generation. The consensus prevents double-spending of digital cash, and the digital cash incentivizes maintenance of the consensus system. More recently, Kroll et al. [2] note that miners' following the "Longest Chain Rule" should be a Nash equilibrium. Biais et al. [3] formalize the mining game and discuss multiple equilibria.1 
Instead of focusing on Bitcoins et al. [4] and Saleh [5] take a holistic approach to examine universal features of blockchains, with a direct focus on how the information distribution that comes with decentralization interacts with the quality of consensus generation, and how proof- of-stake can potentially better incentivize the maintenance of the consensus system.

Importantly, several studies point out considerable challenges against decentralization. Cong et al. [1] discuss a direct tension between providing decentralized consensus and the distribution of information. Besides, risk sharing constitute a natural force against decentralization. For example, Cong et al. [4] argue that risksharing motives lead to the rise of concentrated mining pools. But they also demonstrate how diversification across pools provides an alternative route for decentralization and how pool fees would help prevent over concentration of mining power.

Among studies on the application and economic impact of the technology, Harvey [6] briefly surveys the mechanics and applications of crypto-finance, especially Bitcoin. Yermack [7] evaluates the potential impacts of the blockchain technology on corporate governance. Complementary to our discussion on smart contracts, [8] empirically document how smart contracts are interpreted and programmed on various blockchain platforms. Cong [1] further adds by examining how consensus generation interact with information asymmetry and affect market competition.

The discussion on blockchain applications is not complete without discussion crypto-currencies and crypto-tokens. The total market cap of crypto-currencies reached over 800 billion dollars at one point in 2017 and have become an debated asset class for investment. Initial coin offerings as a new form of financing startups have also taken the market by storm. To understand these phenomena, a first step is to understand how we value cryptoassets.

Cong Lin William, et al. [9] provides the first dynamic assetpricing model of (crypto-) tokens on (blockchain-based) platforms, that endogenizes dynamic adoption and incorporates network externality. Tokens intermediate peer-to-peer transactions, and their trading creates inter-temporal complementarity among users and generates a feedback loop between token valuation and adoption. Consequently, tokens capitalize future platform growth, accelerate adoption, and reduce user-base volatility. Equilibrium token price increases non-linearly in platform productivity, user heterogeneity, and endogenous network size.

Building on this framework, Cong [10] argues that financing startups using crypto- tokens can facilitate fast adoption and continued advancement, and also incentivize early investors and contributors to enhance initial platform technology via the capital gains channel. Yet such benefits come at the expense of entrepreneur's ability to signal project type through token retention, and shirking under volatile token price. A key regulatory implication is that initial coin offerings must come hand-in-hand with vesting of the entrepreneurs and developers.
Several contemporaneous papers also discuss crypto-asset valuation. Athey [11] emphasizes agents' dynamic learning on a binary technology quality and decision to use bitcoins for money transfer, but does not model user-base externality. Gans [12] is among the earliest studies on platform-specific virtual currencies and users' network effects. Pagnotta [13] studies the asset pricing implications of user- base externality in a static setting, with a focus on Bitcoin and miners' incentives but takes user base as exogenous. Ciaian [14] test quantity theory of money using Bitcoin data without modeling agents' optimal decision to use Bitcoins.

A few papers study cryptocurrencies specifically in the context of initial coin offerings (ICOs). In a two-period setting, Sockin et al. [15] study how households first purchase a indivisible cryptocurrency which serves as membership certificate that enables them to match and trade later. Li et al. [16] demonstrate that staged coin offerings mitigate coordination issues. Catalini et al. [17] study ICOs with tokens as coupons for redeeming products and services from the issuing company. Chod et al. [18] discuss diversification benefits of ICOs while Canidio [19] demonstrates how entrepreneurs derive sineigrage. On the empirical side, Hu et al. [20] document crypto-token returns once issued.

Going forward, blockchain protocol design is a wide open area that requires computer scientists and economists to join force. Can there be more efficient and effective decentralized consensus algorithms? Can there be stable tokens that can compete with other types of money or used as a global currency? How can blockchain be combined with the internet of things to better facilitate trade finance?

In terms of applications. We still understand very little about the role of crypto-tokens. Competition among alternative cryptocurrencies is interesting, and can be related to platform competitions more broadly. Abadi et al. [21] and Cong et al [22] are good examples. Applications in auditing systems, financial market exchanges and clearing, trade finance all seem promising, perhaps after the current episode of wild of cryptocurrency speculation is over [23-28].

\section{Acknowledgement}

I gratefully aknowledge financial support from the National Science Foundation of China (Grant Number: 71503183)

\section{References}

1. Cong Lin William, Zhiguo He (2018) Blockchain disruption and smart contracts. p. 51.

2. Kroll JA, Ian C Davey, Edward W Felten (2013) The Economics of Bitcoin Mining, or Bitcoin in the Presence of Adversaries.

3. Biais Bruno, Christophe Bisiere, Matthieu Bouvard, Catherine Casamatta (2018) The blockchain folk theorem. Working Paper pp. 17-817.

4. Cong Lin William, Zhiguo He, Jiasun Li (2018) Decentralized mining in centralized pools. Working Paper.

5. Saleh Fahad (2017) Blockchain Without Waste: Proof-of-Stake, Discussion Paper. Working Paper. 
6. (2016) Economic Analysis of the Digital Economy. In: Harvey, Campbell R, Cryptofinance, University of Chicago Press, USA, pp. 257-276.

7. Yermack David (2017) Corporate governance and blockchains. Review of Finance Forthcoming. p. 47.

8. Bartoletti M, Livio Pompianu (2017) An empirical analysis of smart contracts: Plat- forms, applications, and design patterns. In: Michael Brenner, Kurt Rohloff, Joseph Bonneau, Andrew Miller, Peter Y.A. Ryan, et al. (Eds.), Financial Cryptography and Data Security Cham. Springer International Publishing pp. 494-509.

9. Cong Lin William, Ye Li, Neng Wang (2018) Tokenomics: Dynamic Adoption and Valuation. Working Paper, p. 18-46.

10. Cong Lin William, Ye Li (2018) Tokenomics: Coin offerings, Business acceleration, and Frauds. Working Paper

11. Athey Susan, Ivo Parashkevov, Vishnu Sarukkai, Jing Xia (2016) Bitcoin pricing, adoption, and usage: Theory and evidence. p. 16-42.

12. Gans JS, Hanna Halaburda (2015) Some economics of private digital currency. In: Avi Goldfarb, Shane M Greenstein, Catherine E. Tucker (Eds.), University of Chicago Press, USA, p. 257-276.

13. Pagnotta E, Andrea Buraschi (2018) An Equilibrium Valuation of Bitcoin and Decentralized Network Assets. p. 40.

14. Ciaian, Pavel, Miroslava Rajcaniova, d'Artis Kancs (2016) The Economics of Bitcoin price formation. Applied Economics 48(19): 1799-1815.

15. Sockin, Michael, Wei Xiong (2018) A Model of Cryptocurrencies.

16. Li Jiasun, William Mann (2018) Initial Coin Offering and Platform Building. Working Paper p. 56.

17. Christian C, Joshua S Gans (2018) Initial Coin Offerings and The Value of Crypto Tokens. National Bureau of Economic Research Working Paper pp. 24418.
18. Chod Jiri, Evgeny Lyandres (2018) A theory of ICOs: Diversification, Agency, and Information Asymmetry.

19. Canidio Andrea (2018) Financial incentives for open source development: the case of blockchain. 159270: 85719.

20. Hu Albert, Christine Parlour, Uday Rajan (2018) Cryptocurrencies: Stylized Facts on a New Investible Instrument. Working Paper.

21. Abadi, Joseph, Markus Brunnermeier (2018) Blockchain economics. Working Paper.

22. Cong Lin William, Neng Wang (2018) Tokenomics: Platform competitions. Working Paper.

23. David E, Maureen O'Hara, Soumya Basu (2017) From Mining to Markets: The Evolution of Bitcoin Transaction Fees. p. 48.

24. Eyal I, Emin Gün Sirer (2014) Majority is not Enough: Bitcoin Mining is Vulnerable.

25. Huberman Gur, Jacob D Leshno, Ciamac C Moallemi (2017) Monopoly without a Monopolist: An Economic Analysis of the Bitcoin Payment System. Working Paper.

26. Khapko Mariana, Marius Zoican (2017) Smart Settlement. Working Paper. p. 17-24.

27. Malinova Katya, Andreas Park (2016) Market Design for Trading with Blockchain Technology. p. 40.

28. Nayak Kartik, Srijan Kumar, Andrew Miller, Elaine Shi (2016) Stubborn Mining: Generalizing Selfish Mining and Combining with an Eclipse Attack.
Creative Commons Attribution 4.0 International License

For possible submissions Click Here
Submit Article

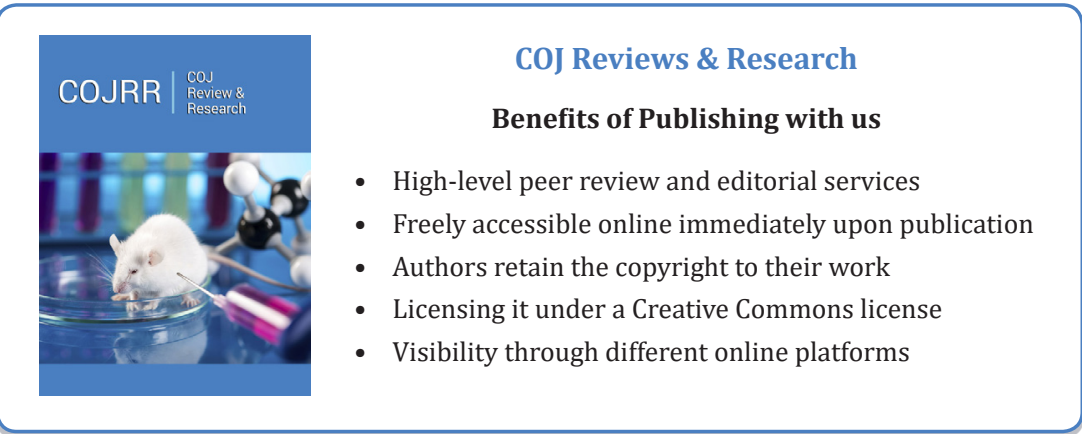

